$\Phi=$

\title{
Squamous cell carcinoma (SCC) of the renal pelvis following chronic nephrolithiasis; presented with feature suggesting xanthogranulomatous pyelonephritis
}

\author{
Mosab Abdalla Ali Alzubier ${ }^{1 *}$, Ahmed Eltyeb Ibrahim ${ }^{2}$, Sami Mahjoub Taha ${ }^{3}$ \\ ${ }^{1}$ Assistant Professor of Urology, MBBS, MRCS, EF urology, Gadarif University, Faculty of Medicine, Sudan. Temporally Assistant \\ Professor of Urology, Prince Sattam ibn Abdulaziz University, Faculty of Medicine, Al kharj, KSA \\ *Corresponding author E-mail: mosab19103@ hotmail.com \\ 2 Consultant Urologist, Department of Urology, Khartoum Teaching Hospital, Sudan \\ 3 Professor of Urology, University of Gezira, Faculty of Medicine, Sudan
}

\begin{abstract}
Background: Squamous cell carcinoma (SCC) which originated in the urothelium of the urological system is not uncommon; however, it is rare in the renal pelvis. Additionally, it is hard to be diagnosed without high clinical suspicion.

Case summary: A-66-years old female presented with left loin pain for 6 years, hematuria, and fever for months. Examination revealed no abnormality apart from pallor and bimanually palpable left kidney. Her investigations showed normal renal function and anemia, Computer Topography for Kidney, Ureter, and Bladder (CT-KUB) revealed a large left renal pelvic stone with a hugely enlarged kidney with suspicion of xanthogranulomatous pyelonephritis (XGPN), with almost absent function of the left kidney in DTPA. She underwent Left trans-peritoneal radical nephrectomy. Post-operative histopathology result was moderately differentiated squamous cell carcinoma (T3, N0, M0). To our knowledge this was the first reported case in our hospital, we think that the treatment was fair putting in mind the poor prognosis of the condition and the difficulty in diagnosis.

Conclusion: This case demonstrates renal pelvis SCC presented with radiological features suggestive of xanthogranulomatous pyelonephritis.
\end{abstract}

Keywords: Squamous Cell Carcinoma; Renal Pelvis; Nephrectomy; Nephrolithiasis; Case Report.

\section{Introduction}

Squamous cell carcinoma of the renal pelvis is a rare disease that accounts for $6-15 \%$ of all upper urinary tract cancers (1). If not suspected clinically, diagnosis of the disease will be difficult and late, hence patients present at advanced stages resulting in poor prognosis (2). There are a lot of factors that predict squamous cell carcinoma in the renal collecting system such as nephrolithiasis, irritation, and chronic inflammation. Nephrolithiasis, especially the formation of staghorn calculi, is the most common risk factor for SCC, which usually occurs in older adults (age 50-65 years) with no sex predilection (3).

\section{Case presentation}

A-66-years old female from the west of Sudan, presented complaining of left loin pain for 6 years and attacks of hematuria for 5 months, also she has many attacks of fever. She has been diagnosed with hypertension for the last 10 years. Her current medication is amlodipine $5 \mathrm{mg}$ once/day and aspirin $100 \mathrm{mg}$ once/day. There was no history of loss of appetite or significant weight loss. Clinical Examination revealed no abnormality apart from pallor and bimanually palpable left kidney. Investigations revealed normal renal function and anemia $(\mathrm{Hb}=8,5 \mathrm{~g} / \mathrm{dl})$, urine the analysis was negative for hematuria, CT-KUB showed large left renal pelvic stone with hugely enlarged kidney with suspicion of xanthogranulomatous pyelonephritis (Fig. 1), with the almost absent function of the left kidney (1\% the split function of the left kidney in DTPA). The patient transfused 2 units of blood and prepared for surgery. Left trans-peritoneal radical nephrectomy was done (midline abdominal incision) as the possibility of malignancy cannot be excluded, the operation was difficult due to massive adhesions and loss of normal architecture of the kidney and surrounding tissues (Fig. 2), the postoperative course was unremarkable, she was discharged in good condition on 5th postoperative day. Histopathology result was moderately differentiated squamous cell carcinoma of the renal pelvis with the involvement of the perinephric fat and no evidence of vascular or lymph node invasion (T3, N0, M0). The patient referred to the MDT clinic in oncology hospital for further management, the opinion was to receive adjuvant chemotherapy because the option of completing the surgery was difficult and risky because of the patient age and comorbidity. Unfortunately, the patient passed away in the oncology hospital after four months. 


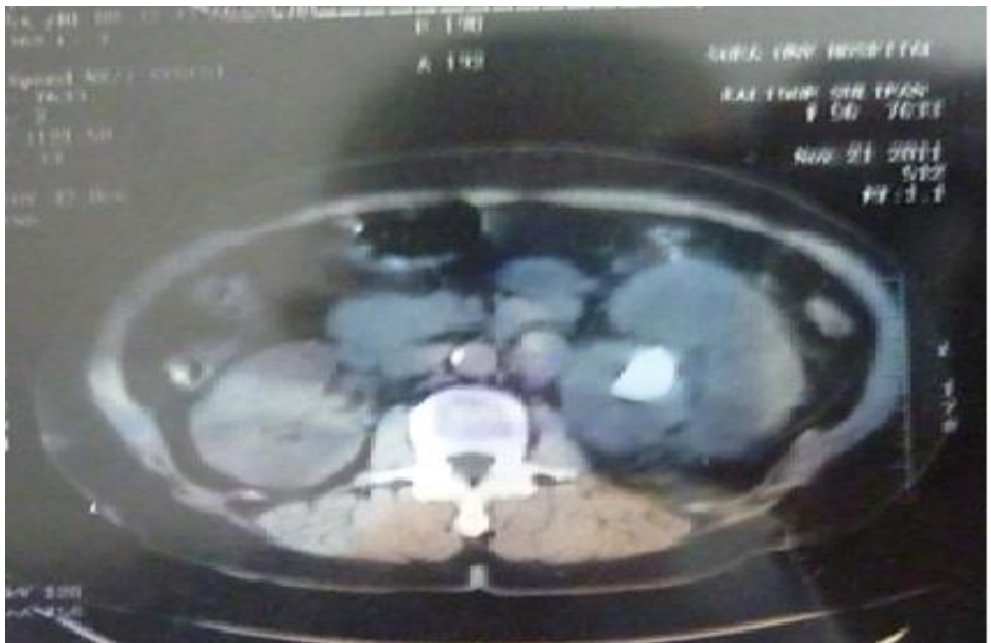

Fig. 1: CT Showing Enlarged Left Kidney with Pelvic Stone, Cystic Changes and Parenchymal Loss.

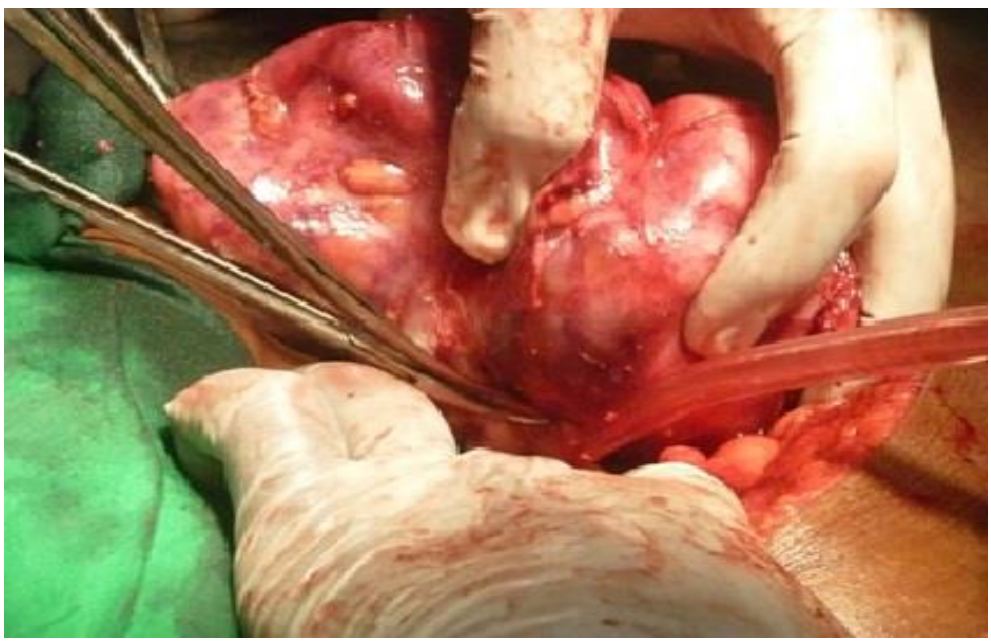

Fig. 2: Intraoperative Appearance of the Left Kidney.

\section{Discussion}

Primary malignant tumors of the renal pelvis are relatively rare and represent 8-14\% of all renal malignancies (4)(5), and 5-6 \% of all urothelial tumors of the urinary tract (1). About $90 \%$ of the urothelial tumors of the renal pelvis and collecting system are transitional cell carcinoma (TCC) (5). Primary squamous cell carcinoma (SCC) of the renal pelvis accounts for about 6-15\% of all upper tract urothelial cancers (1) (0,5-0,8\% of all malignant renal tumors) (6)(7). SCC of the urinary tract is more frequently reported in the urinary bladder and urethra than in the upper urinary tract (6).

Long-standing urolithiasis with chronic infection and inflammation believed to be the most important etiological factor (2), other risk factors include, endogenous and exogenous chemicals, radiation therapy, hormonal imbalance, immune suppression with azathioprine, chronic rejection of transplanted kidney, chronic analgesic abuse, and smoking (4)(7)(3). SCC of the renal pelvis is an aggressive tumor of poor prognosis, usually discovered in late stage due to the difficulty in differentiation from other conditions of the kidney such as xanthogranulomatous pyelonephritis (XGPN) because they have the same etiological factors, clinical presentation (pain, hematuria, recurrent or persistent UTI) (2)(6). This overlap in presentation with a lack of specific radiological features made a preoperative diagnosis of SCC extremely difficult (1), this scenario was typically applied to this case regarding the risk factor, the chronicity of the case, the presenting symptoms, and the differential diagnosis. Radiologically it may appear as a solid mass with hydronephrosis, calcification, or renal pelvic infiltration (6). The treatment of choice for renal pelvic SCC is radical nephroureterectomy with excision of bladder cuff, usually nephrectomy was done to control the symptoms, eliminate the source of infection, and to establish the diagnosis (3), in our case, we did nephrectomy typically to eliminate the bother symptoms putting in our mind the possibility of renal cancer. Chemoradiotherapy in spite of their failure to show survival benefit are still used, the overall median survival is 10 months in comparison to 63 months for TCC (2)(5).

XGPN is an unusual variant of chronic poly-bacterial pyelonephritis with aggressive inflammatory response classically predisposed by urinary tract obstruction and nephrolithiasis resulted in renal parenchymal destruction (6)(8)(9). Usually, it is misdiagnosed as renal cancer, and treated by nephrectomy with or without preoperative nephrostomy and antibiotics (9).

\section{Conclusion}

SCC of the renal pelvis is a relatively rare tumor of insidious presentation, poor prognosis, and dismal outcome. The only possible way to improve the prognosis is early diagnosis and treatment which need a high index of suspicion. It should be suspected in any patient with long-standing renal stone, this emphasizes the necessity of prompt treatment of renal stone and assessment for tumors in patients with long-standing renal stones. 


\section{Abbreviations}

SCC; Squamous Cell Carcinoma. CT-KUB; Computer Topography for Kidney, Ureter and Bladder. XGPN; Xanthogranulomatous pyelonephritis. DTPA; diethylene triamine penta-acetic acid. TCC; Transitional Cell Carcinoma

\section{Consent for publication}

Written informed consent was obtained from the patient for publication of this case report and any accompanying images. The journal is authorized to publish this case.

\section{Competing interests}

Authors declare no competing interest

\section{Funding}

No funding was received for this study.

\section{Authors' contributions}

MAA and AAI were the treating physician of the patient. MAA and SMT designed the study and drafted the manuscript. All authors read and approved the final manuscript.

\section{Acknowledgements}

Not applicable.

\section{References}

[1] Jain A, Mittal D, Jindal A, Solanki R, Khatri S, Parikh A, et al. Incidentally Detected Squamous Cell Carcinoma of Renal Pelvis in Patients with Staghorn Calculi: Case Series with Review of the Literature. ISRN Oncol. 2011; 2011:1-6. https://doi.org/10.5402/2011/620574

[2] Geng JH, Chen YT, Juan YS, Huang CH, Chou YH, Wang CJ, et al. Incidentally detected squamous cell carcinoma of the renal pelvis in patients with staghorn calculi - A case report and literature review. Urol Sci. 2013; 24(4):136-9. https://doi.org/10.1016/j.urols.2013.05.004

[3] Singh V, Sinha RJ, Sankhwar SN, Mehrotra B, Ahmed N, Mehrotra S. Squamous Cell Carcinoma of the Kidney - Rarity Rede fi ned : Case Series with Review of Literature. 2010; 2(4):82-5.

[4] Attalla K, Haines K, Labow D, Mehrazin R. Squamous Cell Carcinoma of the Renal Pelvis: Atypical Presentation of a Rare Malignancy. Urol Case Reports [Internet]. 2017; 13:137-9. Available from: https://doi.org/10.1016/j.eucr.2017.04.007.

[5] Report C. Clinics in Surgery Squamous Cell Carcinoma of the Renal Pelvis Presented as Post-Obstruction Pyonephrosis : A Commonly Known Malignancy in a Rare Location. 2019; 4:4-6.

[6] Kalayci O, Bozdag Z, Sonmezgoz F, Sahin N. Squamous cell carcinoma of the renal pelvis associated with kidney stones: Radiologic imaging features with gross and histopathological correlation. J Clin Imaging Sci. 2013; 3(1):1-4. https://doi.org/10.4103/2156-7514.109741.

[7] Kim JR, Jeong YB, Lee NH, Wang S IL. Squamous cell carcinoma of the renal pelvis presenting as an integumentary neoplasm of the flank: A case report. Med (United States). 2019; 98(36):2-6. https://doi.org/10.1097/MD.0000000000017049.

[8] Ho CI, Wen YK, Chen ML. Xanthogranulomatous pyelonephritis successfully treated with antibiotics only. J Chinese Med Assoc. 2008; 71(12):643-5. https://doi.org/10.1016/S1726-4901(09)70008-5.

[9] Ramos LD, De M, Lima M, De Carvalho M, Bezerra G, Júnior S, et al. Emphysematous and xanthogranulomatous pyelonephritis: rare diagnosis. Vol. 14, Braz J Infect Dis. 2010. https://doi.org/10.1590/S1413-86702010000400011. 